OITS-538

\title{
Hadronic Penguin B Decays In The Standard And The Two-Higgs-Doublet Models *
}

\author{
N.G. Deshpande and Xiao-Gang He \\ Institute of Theoretical Science \\ University of Oregon \\ Eugene, OR 97403-5203, USA
}

(March, 1994; Revised May, 1994)

\begin{abstract}
We study in next-to-leading order QCD hadronic penguin $B$ decays in the Standard and two-Higgs-doublet models. Although the gluonic penguin dominates, we find the electroweak contribution non-negligible. In the Standard Model, the branching ratio for $B \rightarrow X_{s} \phi$ is predicted to be in the range $(0.6 \sim 2) \times 10^{-4}$. The ranges of branching ratios for $B \rightarrow K \phi, B \rightarrow K^{*} \phi$, and $B_{s} \rightarrow \phi \phi$ are $(0.4 \sim 2) \times 10^{-5},(0.2 \sim 1) \times 10^{-5}$, and $(0.15 \sim 0.5) \times 10^{-5}$, respectively. The contribution from the charged Higgs boson in two Higgs doublet models depend on $\cot \beta$, and can be as large as $40 \%$.
\end{abstract}

Typeset using REVTEX

*Work supported in part by the Department of Energy Grant No. DE-FG06-85ER40224. 
Rare $B$ decays, particularly pure penguin decays, have been subject of considerable theoretical and experimental interest recently [1]. The photonic penguin induced process $B \rightarrow K^{*} \gamma$ has been observed by CLEO collaboration [2] and is consistent with the Standard Model (SM) prediction [3]. The gluonic penguin induced $B$ decays are expected to be observed very soon. A large number of gluonic penguin induced $B$ decay channels were studied in Ref. [4] using $\Delta B=1$ effective Hamiltonian $H_{\Delta B=1}$ in the lowest nonvanishing order. In Ref. [5] the next-to-leading order QCD corrected pure gluonic penguin $H_{\Delta B=1}$ was used with top quark mass $m_{t}$ fixed at $150 \mathrm{GeV}$. In this paper we study the next-to-leading order QCD corrected Hamiltonian $H_{\triangle B=1}$ in the SM and in two Higgs doublet models, taking particular care to include the full electroweak contributions and find the dependence on $m_{t}$ and $\alpha_{s}$. Using this Hamiltonian we study the cleanest signature of hadronic penguin processes: $B \rightarrow X_{s} \phi, B \rightarrow K \phi\left(K^{*} \phi\right)$, and $B_{s} \rightarrow \phi \phi$. The process $B \rightarrow X_{s} \phi$ is particularly recommended because it is free from form factor uncertainties. We find not only that the QCD correction in next-to-leading order are large, but also inclusion of the full electroweak contributions have significant effect on the branching ratio which could reduce the pure gluonic penguin contribution by $30 \%$ at the upper range of allowed top quark mass. Our

results which have been derived independently, agree with Ref. [6] where only the SM is considered.

$\Delta B=1$ gluonic penguin Hamiltonian

The QCD corrected $H_{\triangle B=1}$ relevant to us can be written as follows [7]:

$$
H_{\Delta B=1}=\frac{G_{F}}{\sqrt{2}}\left[V_{u b} V_{u s}^{*}\left(c_{1} O_{1}^{u}+c_{2} O_{2}^{u}\right)+V_{c b} V_{c s}^{*}\left(c_{1} O_{1}^{c}+c_{2} O_{2}^{c}\right)-V_{t b} V_{t s}^{*} \sum c_{i} O_{i}\right]+\text { H.C. },
$$

where the Wilson coefficients (WCs) $c_{i}$ are defined at the scale of $\mu \approx m_{b}$; and $O_{i}$ are defined as

$$
\begin{array}{ll}
O_{1}^{q}=\bar{s}_{\alpha} \gamma_{\mu}\left(1-\gamma_{5}\right) b_{\beta} \bar{q}_{\beta} \gamma^{\mu}\left(1-\gamma_{5}\right) q_{\alpha}, & O_{2}^{q}=\bar{s} \gamma_{\mu}\left(1-\gamma_{5}\right) b \bar{q} \gamma^{\mu}\left(1-\gamma_{5}\right) q, \\
O_{3}=\bar{s} \gamma_{\mu}\left(1-\gamma_{5}\right) b \sum_{q^{\prime}} \bar{q}^{\prime} \gamma_{\mu}\left(1-\gamma_{5}\right) q^{\prime}, & Q_{4}=\bar{s}_{\alpha} \gamma_{\mu}\left(1-\gamma_{5}\right) b_{\beta} \sum_{q^{\prime}} \bar{q}_{\beta}^{\prime} \gamma_{\mu}\left(1-\gamma_{5}\right) q_{\alpha}^{\prime}, \\
O_{5}=\bar{s} \gamma_{\mu}\left(1-\gamma_{5}\right) b \sum_{q^{\prime}} \bar{q}^{\prime} \gamma^{\mu}\left(1+\gamma_{5}\right) q^{\prime}, & Q_{6}=\bar{s}_{\alpha} \gamma_{\mu}\left(1-\gamma_{5}\right) b_{\beta} \sum_{q^{\prime}} \bar{q}_{\beta}^{\prime} \gamma_{\mu}\left(1+\gamma_{5}\right) q_{\alpha}^{\prime},
\end{array}
$$




$$
\begin{aligned}
& O_{7}=\frac{3}{2} \bar{s} \gamma_{\mu}\left(1-\gamma_{5}\right) b \sum_{q^{\prime}} e_{q^{\prime}} \bar{q}^{\prime} \gamma^{\mu}\left(1+\gamma_{5}\right) q^{\prime}, \quad Q_{8}=\frac{3}{2} \bar{s}_{\alpha} \gamma_{\mu}\left(1-\gamma_{5}\right) b_{\beta} \sum_{q^{\prime}} e_{q^{\prime}} \bar{q}_{\beta}^{\prime} \gamma_{m} u\left(1+\gamma_{5}\right) q_{\alpha}^{\prime}, \\
& O_{9}=\frac{3}{2} \bar{s} \gamma_{\mu}\left(1-\gamma_{5}\right) b \sum_{q^{\prime}} e_{q^{\prime}} \bar{q}^{\prime} \gamma^{\mu}\left(1-\gamma_{5}\right) q^{\prime}, \quad Q_{10}=\frac{3}{2} \bar{s}_{\alpha} \gamma_{\mu}\left(1-\gamma_{5}\right) b_{\beta} \sum_{q^{\prime}} e_{q^{\prime}} \bar{q}_{\beta}^{\prime} \gamma_{m} u\left(1-\gamma_{5}\right) q_{\alpha}^{\prime}
\end{aligned}
$$

The WCs $c_{i}$ are obtained by solving the renormalization group equation

$$
\left(\mu \frac{\partial}{\partial \mu}+\beta(g) \frac{\partial}{\partial g}\right) \mathbf{C}\left(m_{W}^{2} / \mu^{2}, g^{2}\right)=\hat{\gamma}^{T}\left(g^{2}\right) \mathbf{C}\left(m_{W}^{2} / \mu^{2}, g^{2}\right)
$$

Here $\mathbf{C}$ is the column vector $\left(c_{i}\right)$, and

$$
\begin{gathered}
\beta(g)=-\left(11-\frac{2}{3} n_{f}\right) \frac{g^{3}}{16 \pi^{2}}-\left(102-\frac{38}{3} n_{f}\right) \frac{g^{5}}{\left(16 \pi^{2}\right)^{2}}+\ldots \\
\hat{\gamma}\left(g^{2}\right)=\gamma_{s}^{(0)} \frac{\alpha_{s}}{4 \pi}+\gamma_{s}^{(1)} \frac{\alpha_{s}^{2}}{(4 \pi)^{2}}+\gamma_{e}^{(0)} \frac{\alpha_{e m}}{4 \pi}+\gamma_{s e}^{(1)} \frac{\alpha_{e m} \alpha_{s}}{(4 \pi)^{2}}+\ldots
\end{gathered}
$$

where $n_{f}$ is the number of active quark flavours.

The anomalous-dimension matrix $\gamma_{s}^{(0)}$ and the first term in $\beta(g)$ determine the leading $\log$ QCD corrections [B]. The rest of the terms contain information about the leading QED and next-to-leading order QCD corrections. The full $10 \times 10$ matrices for $\gamma_{i}$ are given in Ref. [7]. The matching conditions of the Wilson coefficients at $m_{W}$ for the next-to-leading order corrections will be different from the leading order ones. One needs to include one loop current-current corrections for $c_{1,2}$ at $m_{W}$. The full results for the initial conditions can be found in [7].

The WCs obtained above depend on the renormalization regularization scheme (RS) used. In our calculation we used the naive dimentional regularization scheme. The physical quantities, of course, should not depend on RS provided one handels the hadronic matrix elements correctly. In practice, many of the hadronic matrix elements can only be calculated using factorization method. In our later calculation we will also use this approxmation. Since this approximation does not carry information about the RS dependence, it is better for us to use WCs, $\overline{\mathbf{C}}(\mu)=\left(\mathbf{1}+\hat{\mathbf{r}}_{\mathbf{s}}^{T} \alpha_{s}(\mu) / 4 \pi+\hat{\mathbf{r}}_{\mathbf{e}}^{T} \alpha_{e m}(\mu) / 4 \pi\right) \mathbf{C}(\mu)$, which are RS independent [9]. Here the matrices $\hat{\mathbf{r}}_{s, e}$ are obtained from one-loop matching conditions. The $6 \times 6 \hat{\mathbf{r}}_{s}$ matrix for the pure gluonic penguin operators has been given in Ref. [9]. Based on the work of Ref. 
[9], we have worked out the full $10 \times 10$ matrices for $\hat{\mathbf{r}}_{s, e}$ and carried out the calculation using the full matrices.

We also need to treat the matrix elements to one-loop leve for consistency. These oneloop matrix elements can be rewritten in terms of the tree-level matrix elements $\left\langle O_{j}\right\rangle^{\text {tree }}$ of the effective operators, and one obtains [6, [10]

$$
<c_{i} O_{i}>=\sum_{i j} c_{i}(\mu)\left[\delta_{i j}+\frac{\alpha_{s}}{4 \pi} m_{i j}^{s}+\frac{\alpha_{e m}}{4 \pi} m_{i j}^{e}\right]<O_{j}>^{\text {tree }} .
$$

We have worked out the full matrices $m^{s, e}$. For the processes we are considering only $\bar{c}_{3-10}$ contribute. Expressing the effective coefficients $c_{i}^{\text {eff }}$ which multiply the matrix elements $<O_{j}>^{\text {tree }}$ in terms of $\bar{c}_{i}$, we have

$$
\begin{aligned}
& c_{3}^{e f f}=\bar{c}_{3}-P_{s} / 3, \quad c_{4}^{e f f}=\bar{c}_{3}+P_{s}, \quad c_{5}^{\text {eff }}=\bar{c}_{5}-P_{s} / 3, \quad c_{6}^{\text {eff }}=\bar{c}_{6}+P_{s}, \\
& c_{7}^{\text {eff }}=\bar{c}_{7}+P_{e}, \quad c_{8}^{\text {eff }}=\bar{c}_{8}, \quad c_{9}^{\text {eff }}=\bar{c}_{9}+P_{e}, \quad c_{10}^{\text {eff }}=\bar{c}_{10} .
\end{aligned}
$$

The leading contributions to $P_{s, e}$ are given by: $P_{s}=\left(\alpha_{s} / 8 \pi\right) \bar{c}_{2}\left(10 / 9+G\left(m_{c}, \mu, q^{2}\right)\right)$ and $P_{e}=\left(\alpha_{e m} / 9 \pi\right)\left(3 \bar{c}_{1}+\bar{c}_{2}\right)\left(10 / 9+G\left(m_{c}, \mu, q^{2}\right)\right)$. Here $m_{c}$ is the charm quark mass which we take to be $1.35 \mathrm{GeV}$. The function $G\left(m, \mu, q^{2}\right)$ is give by

$$
G\left(m, \mu, q^{2}\right)=4 \int_{0}^{1} x(1-x) \mathrm{d} x \ln \frac{m^{2}-x(1-x) q^{2}}{\mu^{2}} .
$$

In the numerical calculation, we will use $q^{2}=m_{b}^{2} / 2$ which represents the average value and the full expressions for $P_{s, e}$.

Using range of values of $\alpha_{s}\left(m_{Z}\right)$ and $m_{t}$ we can calculate the coefficients at $\mu=m_{b}$. We use $\alpha_{s}\left(m_{Z}\right)$ as input instead of $\Lambda_{4}$ as in Ref. [9]. In Table 1, we show some sample WCs for the central world average value of $\alpha_{s}\left(m_{Z}\right)=0.118$ [1] and for several values of $m_{t}$ with $\alpha_{e m}=1 / 128$.

In the two-Higgs-doublet model, there are new contributions to $c_{i}$ due to charged Higgs boson. The charged Higgs-quark couplings are given by [12]

$$
L_{H}=\frac{g}{2 \sqrt{2} m_{W}} \bar{u}_{i} V_{i j}\left[\cot \beta m_{u_{i}}\left(1-\gamma_{5}\right)-a m_{d_{j}}\left(1+\gamma_{5}\right)\right] d_{j} H^{+}+H . C .,
$$


where $\cot \beta=v_{1} / v_{2} ; v_{1}$ and $v_{2}$ are the vacuum expectation values of the Higgs doublets $H_{1}$ and $H_{2}$, which generate masses for down and up quarks, respectively. The parameter $a$ depends on the models [12]. The main contributions are from the first term in eq.(8) and we will neglect the contribution from the second term. The charged Higgs contributions to gluonic penguin have been studied by several groups [13]. The leading QCD corrected Hamiltonian has been given in Ref. [14]. We have checked the next-to-leading initial conditions for the WCs at $m_{W}$. We find that the inclusion of charged Higgs will not change the initial conditions for $c_{1,2,8,10}$, but $c_{3,4,5,6,7,9}$ are changed in the same way as those given in eqs.(32-38) of Ref. [14].

$\mathrm{B} \rightarrow \mathbf{X}_{\mathbf{s}} \phi$

Using $H_{\Delta B=1}$ in eq.(1), we obtain the decay amplitude for $B \rightarrow X_{s} \phi$

$$
A\left(B \rightarrow X_{s} \phi\right) \approx A(b \rightarrow s \phi)=-\frac{g_{\phi} G_{F}}{\sqrt{2}} V_{t b} V_{t s}^{*} \epsilon^{\mu} C \bar{s} \gamma_{\mu}\left(1-\gamma_{5}\right) b,
$$

where $\epsilon^{\mu}$ is the polarization of the $\phi$ particle; $C=c_{3}^{\text {eff }}+c_{4}^{\text {eff }}+c_{5}^{\text {eff }}+\xi\left(c_{3}^{\text {eff }}+c_{4}^{\text {eff }}+c_{6}^{\text {eff }}\right)-$ $\left(c_{7}^{e f f}+c_{9}^{e f f}+c_{10}^{e f f}+\xi\left(c_{8}^{e f f}+c_{9}^{e f f}+c_{10}^{e f f}\right)\right) / 2$ with $\xi=1 / N_{c}$, where $N_{c}$ is the number of colors. The coupling constant $g_{\phi}$ is defined by $<\phi\left|\bar{s} \gamma^{\mu} s\right| 0>=i g_{\phi} \epsilon^{\mu}$. From the experimental value for $\operatorname{Br}\left(\phi \rightarrow e^{+} e^{-}\right)$, we obtain $g_{\phi}^{2}=0.0586 \mathrm{GeV}^{4}$.

The decay rate is, then, given by

$$
\Gamma\left(B \rightarrow X_{s} \phi\right)=\frac{G_{F}^{2} g_{\phi}^{2} m_{b}^{3}}{16 \pi m_{\phi}^{2}}\left|V_{t b} V_{t s}^{*}\right|^{2} C^{2} \lambda_{s \phi}^{3 / 2}\left[1+\frac{3}{\lambda_{s \phi}} \frac{m_{\phi}^{2}}{m_{b}^{2}}\left(1-\frac{m_{\phi}^{2}}{m_{b}^{2}}+\frac{m_{s}^{2}}{m_{b}^{2}}\right)\right],
$$

where $\lambda_{i j}=\left(1-m_{j}^{2} / m_{b}^{2}-m_{i}^{2} / m_{b}^{2}\right)^{2}-4 m_{i}^{2} m_{j}^{2} / m_{b}^{4}$.

We normalize the branching ratio to the semi-leptonic decay of $B \rightarrow X_{c} e \bar{\nu}_{e}$. We have

$$
\operatorname{Br}\left(B \rightarrow X_{s} \phi\right)=B r\left(B \rightarrow X_{c} e \bar{\nu}_{e}\right) \frac{\left|V_{t b} V_{t s}^{*}\right|^{2}}{\left|V_{c b}\right|^{2}} \frac{12 \pi^{2} g_{\phi}^{2} \lambda_{s \phi}^{3 / 2}}{m_{\phi}^{2} m_{b}^{2} \rho \eta} C^{2}\left[1+\frac{3}{\lambda_{s \phi}} \frac{m_{\phi}^{2}}{m_{b}^{2}}\left(1-\frac{m_{\phi}^{2}}{m_{b}^{2}}+\frac{m_{s}^{2}}{m_{b}^{2}}\right)\right] .
$$

In the above expression, $\rho=1-8 r^{2}+8 r^{6}-r^{8}-24 r^{4} \ln r$ with $r=m_{c} / m_{b}$, is the phase factor, and $\eta$ is the QCD correction factor in $B \rightarrow X_{c} e \bar{\nu}_{e}$, respectively. We will use $\rho=0.5, \eta=$ 0.889 [15], and the approximation $\left|V_{t b} V_{t s}^{*} / V_{c b}\right|^{2}=1$. The branching ratio $\operatorname{Br}\left(B \rightarrow X_{c} e \bar{\nu}_{e}\right)$ is measured to be 0.108 [16]. 


\section{Exclusive decays $B \rightarrow K \phi\left(K^{*} \phi\right)$ and $B_{s} \rightarrow \phi \phi$}

For the exclusive decays, we will use the factorization method. We have

$$
\begin{gathered}
A\left(B \rightarrow K\left(K^{*}\right) \phi\right)=-\frac{G_{F}}{\sqrt{2}} V_{t b} V_{t s}^{*} C<K\left(K^{*}\right)\left|\bar{s} \gamma_{\mu}\left(1-\gamma_{5}\right) b\right| B><\phi\left|\bar{s} \gamma^{\mu} s\right| 0>, \\
A\left(B_{s} \rightarrow \phi \phi\right)=-\frac{G_{F}}{\sqrt{2}} V_{t b} V_{t s}^{*} C<\phi\left|\bar{s} \gamma_{\mu}\left(1-\gamma_{5}\right) b\right| B_{s}><\phi\left|\bar{s} \gamma^{\mu} s\right| 0>.
\end{gathered}
$$

We can parametrize the matrix elements as

$$
\begin{aligned}
<K\left|\bar{s} \gamma_{\mu}\left(1-\gamma_{5}\right) b\right| B> & =f^{+}\left(q^{2}\right)\left(p_{\mu}^{B}+p_{\mu}^{K}\right)+f^{-}\left(q^{2}\right) q_{\mu} \\
<v\left|\bar{s} \gamma_{\mu}\left(1-\gamma_{5}\right) b\right| B> & =2 V\left(q^{2}\right) i \epsilon_{\mu \nu \lambda \sigma} \epsilon_{v}^{\nu} p^{v \lambda} p^{B \sigma} \\
& +A_{1}\left(q^{2}\right)\left(m_{v}^{2}-m_{B}^{2}\right) \epsilon_{v}^{\mu}-A_{2}\left(q^{2}\right) \epsilon_{v} \cdot q\left(p_{\mu}^{B}+p_{\mu}^{v}\right),
\end{aligned}
$$

where $v$ is a vector meson particle and $\epsilon_{v}^{\mu}$ its polarization. For $B \rightarrow K \phi, q=p^{B}-p^{K}$, and for $B \rightarrow v \phi, q=p^{B}-p^{v}$.

In terms of the form factors defined above, we obtain the decay rates

$$
\begin{aligned}
\Gamma(B \rightarrow K \phi) & =\frac{G_{F}^{2} f^{+2}\left(m_{\phi}^{2}\right) g_{\phi}^{2} m_{B}^{3}}{32 \pi m_{\phi}^{2}}\left|V_{t b} V_{t s}^{*}\right|^{2} C^{2} \lambda_{K \phi}^{3 / 2} \\
\Gamma(B \rightarrow v \phi) & =\frac{G_{F}^{2} g_{\phi}^{2} m_{B}^{3}}{32 \pi}\left|V_{t b} V_{t s}^{*}\right|^{2} C^{2} \lambda_{v \phi}^{3 / 2}\left[2 V^{2}\left(m_{\phi}^{2}\right)+\frac{3}{\lambda_{v \phi}}\left(1-\frac{m_{v}^{2}}{m_{B}^{2}}\right)^{2} A_{1}^{2}\left(m_{\phi}^{2}\right)-A_{2}^{2}\left(m_{\phi}^{2}\right)\right. \\
& \left.+\frac{1}{4 m_{v}^{2} m_{\phi}^{2}}\left(\left(m_{v}^{2}-m_{B}^{2}\right) A_{1}\left(m_{\phi}^{2}\right)-\left(m_{B}^{2}-m_{v}^{2}-m_{\phi}^{2}\right) A_{2}\left(m_{\phi}^{2}\right)\right)^{2}\right]
\end{aligned}
$$

To finally obtain the branching ratios, we will use two sets of form factors obtained by Bauer et. al. [17] and Casalbuoni et. al. [18]. Note that we have used different normalization for the form factors $V$ and $A_{i}$ from those in Refs. [17.18]. The form factors at $q^{2}=0$ are determined by using relativistic quark model in Ref. [17], and by using chiral and effective heavy quark theory in Ref. [18]. The form factors at $q^{2}=0$ in Ref. [17] are given by: $f_{K \phi}^{+}=0.393, V_{K^{*} \phi}=0.062 \mathrm{GeV}^{-1}, A_{1 K^{*} \phi}=-0.077 \mathrm{GeV}^{-1}$, and $A_{2 K^{*} \phi}=0.056 \mathrm{GeV}^{-1}$. In Ref. [18] the form factors at $q^{2}=0$ are: $f_{K \phi}^{+}=0.509, V_{K^{*} \phi}=0.103 \mathrm{GeV}^{-1}, A_{1 K^{*} \phi}=$ $-0.047 \mathrm{GeV}^{-1}$, and $A_{2 K^{*} \phi}=0.034 \mathrm{GeV}^{-1}$. In Ref. 18 the form factors at $q^{2}=0$ for $B_{s} \rightarrow \phi \phi$ are also calculated. They are: $V_{\phi \phi}=0.102 \mathrm{GeV}^{-1}, A_{1 \phi \phi}=-0.046 \mathrm{GeV}^{-1}$, and $A_{2 \phi \phi}=0.033 \mathrm{GeV}^{-1}$. In both papers, the $q^{2}$ dependence of all the form factors were assumed 
to be of a simple pole type. We will use the pole masses used in Refs. [17, 18]. It is interesting to note that the ratios between the exclusive decays and $B \rightarrow X_{s} \phi$ are independent of the Wilson coefficients. If these ratios can be measured experimentally, they can test the models for the form factors. We obtain

$$
\begin{aligned}
& \frac{\operatorname{Br}(B \rightarrow K \phi)}{\operatorname{Br}\left(B \rightarrow X_{s} \phi\right)}= \begin{cases}0.06, & \text { Ref. }[17] \\
0.1, & \text { Ref. [18 }\end{cases} \\
& \frac{\operatorname{Br}\left(B \rightarrow K^{*} \phi\right)}{\operatorname{Br}\left(B \rightarrow X_{s} \phi\right)}= \begin{cases}0.057, & \text { Ref. [17 } \\
0.029, & \text { Ref. 118 }\end{cases} \\
& \frac{\operatorname{Br}(B \rightarrow \phi \phi)}{\operatorname{Br}\left(B \rightarrow X_{s} \phi\right)}=0.023, \quad \text { Ref. 18 }
\end{aligned}
$$

We show in, Fuigure 1, the predictions for the branching ratio $\operatorname{Br}\left(B \rightarrow X_{s} \phi\right)$ in the $\mathrm{SM}$ as a function of top quark mass $m_{t}$ and the strong coupling constant $\alpha_{s}\left(m_{Z}\right)$. The QCD corrections turn out to be important which enhance the branching ratios by about $30 \%$ compared with those of without QCD corrections. There is a large uncertainty in the branching ratios due to error in $\alpha_{s}\left(m_{Z}\right)$. From Figure 1, we see that the error in $\alpha_{s}\left(m_{Z}\right)$ can induce an uncertainty of a factor 2 .

The dominant contribuitons are from the gluonic penguin. There is a very small $m_{t}$ dependence for the branching ratio calculated without the inclusion of the electroweak penguin contributions. The inclusion of the full electroweak contribuitons have sizeable effects which reduce the branching ratios by about $20 \%$ to $30 \%$ for the central value of $\alpha_{s}$ with $m_{t}$ varying from $100 \mathrm{GeV}$ to $200 \mathrm{GeV}$. It is clear from Figure 1 that the full contribution has a large $m_{t}$ dependence.

There may be corrections to the branching ratios predicted by the factorization method. It is a common practice to parameterize the possible new contributions by treating $\xi$ as a free parameter 17 19. Using experimental values from non-leptonic $B$ decays, it is found that [18], $a_{1}=c_{2}+\xi c_{1}$ and $a_{2}=c_{1}+\xi c_{2}$ have the same signs, and $\left|a_{2}\right| \approx 0.27$ and $\left|a_{1}\right| \approx 1.0$. We see that $\xi$ is close to $1 / 2$. To see the effect of varying $\xi$, we plot the predictions for the 
branching ratios for $\xi=1 / 2$ and $\xi=1 / 3$. The branching ratios for $\xi=1 / 2$ are about 2 times those for $\xi=1 / 3$.

For the central value of $\alpha_{s}\left(m_{Z}\right)$ and the central value of $m_{t}=174 \mathrm{GeV}$ reported by $\mathrm{CDF}$ [20], the value for $\operatorname{Br}\left(B \rightarrow X_{s} \phi\right)$ is about $1.7 \times 10^{-4}$ for $\xi=1 / 2$. The exclusive branching ratios $B \rightarrow K \phi$ and $B \rightarrow K^{*} \phi$ are about the same which are $1 \times 10^{-5}$ if the form factors from Ref. [17] are used. If the form factors from Ref. [18 are used, one obtains $\operatorname{Br}(B \rightarrow K \phi) \approx 1.7 \times 10^{-5}, \operatorname{Br}\left(B \rightarrow K^{*} \phi\right) \approx 0.5 \times 10^{-5}$, and $\operatorname{Br}\left(B_{s} \rightarrow \phi \phi\right) \approx 0.4 \times 10^{-5}$.

In Figure 2, we show the ratio of the branching ratios $\operatorname{Br}\left(B \rightarrow X_{s} \phi\right)_{2 H}$ and $\operatorname{Br}(B \rightarrow$ $\left.X_{s} \phi\right)_{S M}$ predicted by the two Higgs doublet model and the SM as a function of $\cot \beta$ for $m_{t}=174 \mathrm{GeV}$ and different values of $m_{H}$ with $\xi=1 / 2$. The depence on $\xi$ is small. From Figure 2, we see that the effects of the charged Higgs boson contributions are small for $\cot \beta<1$. When increasing $\cot \beta$, the charged Higgs contributions become important and the effect is to cancel the SM contributions. When $\cot \beta$ becomes very large the charged Higgs boson contributions become the dominant ones. However, using the information from $B \rightarrow X_{s} \gamma$, it is found that for small $m_{H} \sim 100 \mathrm{GeV}$ and $m_{t} \sim 174 \mathrm{GeV}, \cot \beta$ is constrained to be less than 1 [21]. For these values, the charged Higgs boson effects on the processes discussed in this paper are less than 10\%. For $m_{H} \sim 500 \mathrm{GeV}$, the charged Higgs boson effects can reduce the hadronic penguin $B$ decays by $40 \%$ because the range of $\cot \beta$ allowed from $b \rightarrow s \gamma$ is now larger [21]. The effects become smaller for larger $m_{H}$.

The analyses carried out in this letter can be generalized to other hadronic $B$ decays. We will present the full calculations for the Wilson coefficeints, the full expressions for $P_{s, e}$ and other related decays in a forthcoming paper [22].

We thank Buras, McKellar, Fleischer for useful corespondences and thank Lautenbacher for many useful discussions. 


\section{REFERENCES}

[1] For a review see: B Decays, edited by S. Stone, World Scientific, 1992.

[2] CLEO Collaboration, Phys. Rev. Lett. 71, 674(1993).

[3] N.G. Deshpande et. al., Phys. Rev. Lett. 59, 183(1987); S. Bertolini, F. Borzumati and A. Masiero, ibid, 180(1987); N.G. Deshpande, P. Lo and J. Trampetic, Z. Phys.C40, 369(1988); C. Dominguez, N. Paver and Riazuddin, Phys. Lett. B214, 459(1988); A. Ovchinnikov and V. Slobodenyuk, Phys. Lett. B237, 569(1990); P.J. O'Donnel and H.K.K. Tung, Phys. Rev.D44, 741(1991); R. Casalbuoni et. al., Phys. Lett. B312, 315(1993).

[4] N.G. Deshpande and J. Trampetic, Phys. Rev. D41, 895(1990).

[5] A. Deandrea, et. al., Phys. Lett. B320, 170(1993).

[6] R. Fleischer, Preprint, TUM-T31-40/93 (Z. Phys. in press).

[7] A. Buras, M. Jamin, M. Lautenbacher and P. Weisz, Nucl. Phys. B400, 37(1993); A. Buras, M. Jamin and M. Lautenbacher, ibid, 75(1993); M. Ciuchini, E. Franco, G. Martinelli and L. Reina, Nucl. Phys. B415, 403(1994).

[8] F. Gilman and M. Wise, Phys. Rev. D20, 2392(1979); R. Miller and B. McKellar, Phys. Rept. 106, 169(1984).

[9] A. Buras, M. Jamin, M. Lautenbacher and P. Weisz, Nucl. Phys. B370, 69(1992).

[10] R. Fleischer, Z. Phys. C58, 483(1993); G. Kramer, W. Palmer and H. Simma, Preprint, DESY-93-192.

[11] S. Bethke, in Proceedings of the XXV International Conference on High Energy Physics, Dallas, Texas, August, 1992.

[12] J. Gunion, H. Haber, G. Kane and S. Dawson, The Higgs Hunter's Guide (AddisonWesley, Redwood City, CA 1990). 
[13] Wei-Shu Hou and R.S. Willey, Phys. Lett. B202,591(1988); Wei-Shu Hou, Nucl. Phys. B326, 54(1989); V. Barger, J. Hewett and R. Phillips, Phys. Rev. D41, 3421(1990); A. Davies, G.C. Joshi and M. Matsuda, Z. Phys. C52,97(1991); A. Davies, T. Hayashi, M. Matsuda and M. Tanimoto, Preprint, AUE-02-93.

[14] G. Buchalla, A. Buras, M. Harlander, M. Lautenbacher and C. Salazar, Nucl. Phys. B355, 305(1991).

[15] N. Cabbibo and L. Maiani, Phys. Lett. B79, 109(1978); M. Suzuki, Nucl. Phys. B145, 420(1978); N. Cabbibo, G. Corbe and L. Maiani, Phys. Lett. B155, 93(1979).

[16] P. Drell, in Proceedings of the XXV International Conference on High Energy Physics, Dallas, Texas, August, 1992.

[17] M. Bauer, B. Stech and M. Wirbel, Z. Phys. C34, 103(1087).

[18] A. Deandrea, N. Di Bartolomeo, R. Gatto and G. Nardulli, Phys. Lett. B318, 549(1993).

[19] N.G. Deshpande, M. Gronau and D. Sutherland, Phys. Lett. B90, 431(1980).

[20] F. Abe, et al., CDF Collaboration, Preprint, FERMILAB-PUB-94/097-E, CDF/PUB /TOP/PUBLIC/2561.

[21] J.L. Hewett, Phys. Rev. Lett. 70, 1045(1993); V. Barger, M. Berger, R. Phillips, ibid, 1368(1993).

[22] N. Deshpande and Xiao-Gang He, in preparation. 


\section{TABLES}

TABLE I. The Wilson coefficients for $\Delta B=1$ at $m_{b}=5 \mathrm{GeV}$ with $\alpha_{s}\left(m_{Z}\right)=0.118$.

\begin{tabular}{c|c|c|c|c|c|c|c|c|c|c|}
\hline \hline$m_{t}(\mathrm{GeV})$ & $\bar{c}_{1}$ & $\bar{c}_{2}$ & $\bar{c}_{3}$ & $\bar{c}_{4}$ & $\bar{c}_{5}$ & $\bar{c}_{6}$ & $\bar{c}_{7} / \alpha_{e m}$ & $\bar{c}_{8} / \alpha_{e m}$ & $\bar{c}_{9} / \alpha_{e m}$ & $\bar{c}_{10} / \alpha_{e m}$ \\
\hline 130 & -0.313 & 1.150 & 0.017 & -0.037 & 0.010 & -0.045 & -0.061 & 0.029 & -0.978 & 0.191 \\
\hline 174 & -0.313 & 1.150 & 0.017 & -0.037 & 0.010 & -0.046 & -0.001 & 0.049 & -1.321 & 0.267 \\
\hline 210 & -0.312 & 1.150 & 0.018 & -0.038 & 0.010 & -0.046 & 0.060 & 0.069 & -1.626 & 0.334 \\
\hline \hline
\end{tabular}




\section{Figure Captions}

Figure 1. $\operatorname{Br}\left(B \rightarrow X_{s} \phi\right)$ as a function of $m_{t}$ and $\alpha_{s}\left(m_{Z}\right)$. The regions between the dashed and solid lines are the branching ratios for $\alpha_{s}\left(m_{Z}\right)$ varying from 0.111 to 0.125 for $\xi=1 / 2$ and $\xi=1 / 3$, respectively. The branching ratios increases with $\alpha_{s}\left(m_{Z}\right)$.

Figure 2. $\operatorname{Br}\left(B \rightarrow X_{s} \phi\right)_{2 H} / \operatorname{Br}\left(B \rightarrow X_{s} \phi\right)_{S M}$ as a function of $\cot \beta$, and $m_{H}$. The curves 1, 2 and 3 are for $m_{H}$ equals to 100,500 and $1000 \mathrm{GeV}$, respectively. 
This figure "fig1-1.png" is available in "png" format from: http://arxiv.org/ps/hep-ph/9403266v3 